\title{
Evaluation of State University and College Institutional Websites in the Philippines
}

Randy Joy Magno Ventayen*

Pangasinan State University, Philippines; rventayen@psu.edu.ph

\begin{abstract}
Objectives: A quality website provides quality information. In this paper, it investigated and evaluated the State University and Colleges in the Philippines. The main objective of this study is to measure the overall performance of the website regarding accessibility, experience, marketing, and technology. This paper also investigates how the Pangasinan State University ranks with other SUC in the Philippines. Methods/Statistical Analysis: The web address from all state universities and colleges in the Philippines, there are 114 SUC in the Philippines that was tested based on the four categories of accessibility, experience, marketing, and technology. Nibbler was used to testing all the websites and scored from 0 as the lowest to 10 as the highest. The underlying mathematical formula was used to determine the overall rating. Sorting was used to determine the ranking of each website compared to another website. Findings: The result shows that University of the Philippines and Visayas State University ranked first in the list of 114 total number of SUC. While one focus of this study is to determine the ranking of Pangasinan State University, the overall position of the institution was ranked at fifth place. The result of the study shows that SUC website in the Philippines provided a satisfying result. The Top 5 institutional websites based on the testing tool was known as the institutions with a high number of Center of Excellence and Center of Development. Pangasinan State University is doing well in web rankings toowith an overall score of 8.3 making in the $5^{\text {th }}$ place nationwide from a previous score of 4.9 in the year 2015. Lastly, this evaluation provides a quality measure to all government institution website. Application/Improvements: The evaluation of the SUC Website will help the researchers and web administrators to propose a quality measure for an institution website.
\end{abstract}

Keywords: Institution, Philippines, Ranking, Website

\section{Introduction}

The world is connected online in a global system of interconnected computers connected that consist of public and private, academic, business and government clients. The technology aims to minimize the workload and maximize productivity. Developers and designers aim for better technological advancement to improve the way of living. There are several advancements happened in the recent years, such as the improvement of Web services, from web 1.0 to the newly updated web platform. Storage even upgraded into the cloud for more security and productivity. Thus, web services are now growing demand in the market and ranking affects the popularity of the website $\stackrel{1}{\text {. }}$
In recent years, it is visible that there has been an increasing number of technological advantages thru information. Websites were used to provide this information to the humankind. Over the past decade, there has been a dramatic increase in the online application that used by universities to provide better service to its students. Recently, web services and SEO has studied extensively ${ }^{2-3}$. The primary goal of web services in the past was to develop an easier task in the organization such as the institution.

The progress of information and technologies and the birth of the internet changed by the user experience and environment, in advancement way of delivering information and services. It can now be web-enabled, unlike the

*Author for correspondence 
traditional stationary information systems. It is a gateway to information and services from multiple sources in a unified way by using a single and unique user interface. A unit in the university usually manages the web services called the Management Information System (MIS). It is broadly used and applied term for a three-resource system that required effective organization management in the use of information. The resources are people, information, and technology, from inside and outside the organization, with a top priority given to the people. People often use the internet to fasten transactions, communications and easy access to information.

The Pangasinan State University domain psu. edu.ph was registered in domain registrar Philippine Network Foundation, Inc. (PHNET) in 2002 based on the record of the web administration unit. Since then, the website is the primary source of information from the institution.

During the starting days until the recent days, the institutional website was regularly updated to provide the information needs of those who prospective students and other stakeholders of the university. The development of a website could be easier for web developers but maintaining a website especially a corporate website is a big challenge. In July 2016, the rank of PSU. edu.ph website in region 1 is rank 6 out of 6 SUC with a global rank of around 9 million while on April 2017, the institution website jumped to the $2^{\text {nd }}$ place in the region. This call for another study to compare the Pangasinan State University institutional website to the other SUC in the country.

\subsection{Research Objective}

One primary objective of this research is to evaluate the State and University websites across the country to determine the strength and weakness that provides a comparison to the SUC website in the Philippines.

\subsection{Research Questions}

Research question will enable the research to solve the problem. This research sought to answer the following question. What is the primary Educational Institution's Information regarding the URL and the Region? Second, what is the overall performance of the website regarding Accessibility, Experience, Marketing and Technology? Lastly, what is the distribution of SUC regarding overall rating, and what is the Top 5 SUC in the whole country.

\subsection{Scope and Delimitation}

A government institution is called SUC at State University and Colleges; it is an institution mandated by the government. This studyislimited only to theState Universities and Colleges in the Philippines. Local University runs by the LGU and Private Institutions excluded in this study.

The study is limited only in the present assessment of the web services of the institution for the year 2018 . While for the financial information, the data limited to the previous three years.

\subsection{The Significance of the Study}

The significance of this study is for us to identify the strength and weakness of the Philippine institution website. This study does not aim to allow competition between the SUC website across the country. The evaluation of the SUC Website will help the researcher to propose a quality measure for an institution website.

This study has specific significance to the following (1) The State University and Colleges; this will be the bases for the measure on the web services. (2) The University Officials will help them to decide better for their organization and the appointment of web administrator based on the qualification. (3) The Web Administrators will guide them on the standard for the development of the institutional website.

\subsection{Definition of Terms}

The following terms used in this study that defined to understand the overall content of the study.

1. SUC - State Universities and Colleges, it is a government school in tertiary level mandated by the government and governs by the Commission on Higher Education.

2. LGU - Local Government Unit, It is the part of the government closest to the people and is in charge of delivering essential services ${ }^{4}$.

3. MIS - Management Information System, it is the unit of the University that manage the systems that include web services.

4. University Web Administrator - A person in charge of the development and maintaining of the university website. 
5. PSU - Refers to Pangasinan State University, a prestigious institution located at Pangasinan.

6. COE - Center Of Excellence (COE) refers to aninstitution within higher education, which continuously demonstrates excellent performance in the areas of teaching, research that includes publication, extension and linkages and institutional qualifications

7. COD - Center Of Development (COD) refers to aninstitution within higher education, which demonstrates the possibility to become a Center of Excellence in the future.

\subsection{State University and Colleges Web Ranking}

Search engine optimization is one way to increase the popularity of the website ${ }^{5}$, and web developers use this approach to gather customers online such as in the tourism industry ${ }^{6}$. While popularity is not an issue in the SUC websites, it is still needed to determine if the website has quality structure or contribution.

This study is similar to the previous study of the author regarding the web rankings comparison, while in the previous study the scope is limited to region 1, this study investigates the general status of state colleges and universities. In the previous study, the author discusses that the Pangasinan State University which is most commonly known as PSU was previously ranked $6^{\text {th }}$ in the region ${ }^{7}$ to the present ranked of $2^{\text {nd }}$ in the region.

In this study, the researcher tested all SUC websites using Nibbler. It is is a free tool for testing websites and provide a report scoring the website out of 10 for critical areas, including accessibility, SEO, social media ${ }^{8}$ and technology. A total of critical areas that include Facebook page, Twitter, Meta tags, Server behavior, Amount of content, Popularity, Social interest, Internal links, Headings, Images, URL format, Printability, Incoming links, Page titles, Analytics, Mobile, Freshness, and domain age. While there are 17 key areas, these critical areas are divided into four categories which includeaccessibility, experience, marketing,and technology.

\subsection{Accessibility}

Accessibility has answered the word how accessible the website is to mobile and disabled users. The contributing test includes Internal links, Headings, URL format, Page titles and Mobile. Accessibility is an essential factor of a quality website - that contribute to the success in the process of implementing successful websites.

\subsection{Experience}

Experience is about how satisfying the website is likely to be for the users. The contributing test includes a Facebook page, twitter, server behavior, amount of content, popularity, internal links, images, URL format, Printability, Mobile and Freshness.

\subsection{Marketing}

Marketing category determined how well marketed and popular the website is. The contributing test is a Facebook page, twitter, meta tags, amount of content, popularity, social interest, internal links, headings, incoming links, page titles, analytics, and freshness.

\subsection{Technology}

Technology refers to how well designed and built the website is. The contributing tests include Meta tags, server behavior, internal links, headings, images, URL format, printability, and mobile. Technology plays a big part in the quality of the website such as hosting ${ }^{10}$; a high end hosting is the life of a fast website.

\section{Methodology}

This part dealt with the various types of procedures and techniques used by the researchers in gathering relevant information needed to complete the study. It includes the research design, population, and locale of the study, data gathering procedure, data gathering tool, sampling, validity, and treatment of data.

\subsection{Research Design}

The researchers use the descriptive type of method to present and elaborate facts adequately and precisely. It will survey the institutional website of the SUC in the Philippines. The purposes of this research are to find a new truth, to gather information, to classify and to present data based on the data collected thru the assessment of the SUC websites. It will also provide an accurate interpretation of findings according to the standard level/ assessment set by the website standard. 


\subsection{Locale and Population of the Study}

The population of this study is the State and University of the Philippines which includes 8 SUCs in Metro Manila or NCR, 49 in the rest of Luzon, 26 in the Visayas, and 29 in Mindanao. The lists in 2018 of the Commission on Higher Education and the Department of Budget and Management on the grant of free tuition appear to have 114 SUCs in the country.

\subsection{Data Gathering and Testing Procedure}

For the data gathering procedure, the researcher will gather information from the Commission on Higher Education on the profiling of the 114 SUC in the Philippines. If data would be unavailable, a search engine will be utilized to gather data. The Department of Budget and Management additional source of data for the supplemental information of the SUCs.

For extraction of the overall performance of the website, a website evaluation tester nibbler.silktide. com was used to assess every site. The website provided 17 key areas, which are divided into four categories which includeaccessibility, experience, marketing, and technology. Google Chrome browser was used during the testing, while Internet Explorer was usedto revalidating the data that has been gathered.

The tool scored each of the critical areas with 0.0 as the lowest to 10.0 as the highest. 8 to 10 range are made as Excellent, 6 to 7.9 are interpreted as Good, 4 to 5.9 are interpreted as Fair, 2 to 3.9 are interpreted Poor, while 0 to 1.9 is interpreted as Very Poor. All data gathered was manually encoded into a spreadsheet. The underlying mathematical formula was usedto determine the overall rating. VLOOKUP and COUNTIF were used to count and identify the number of SUC based on the excellence. Sorting was usedto determine the ranking of each website compared to another website.

\section{Result and Discussion}

There are 114 SUC tested. The lists came from the 2018 National Expenditure Program and the joint guidelines of the Commission on Higher Education and the Department of Budget and Management ${ }^{11}$. In the total of 114 SUC, there are 11 SUC that either has no website, offline or using gov.ph domain, this issue provided no result for the 11 SUC.
Tables 1,2 shows the first batch out of three batches of the result. The results show that the University of the Philippines ranked first in the National Capital Region with an overall rating of 8.9. In region 1, the Pangasinan State University ranked first with an overall rating of 8.3 compared to the five remaining competitors, while it is visible then there is a close rating with the Mariano Marcos State University with 0.1 difference. In the Cordillera Administrative Region, Mountain Province State University ranked first with an overall rating of 8.4 compared to other SUC in the Region. Nueva Vizcaya State University is the first in the Region 2, with an overall rating of 7.8 compared to other SUC in the region. Lastly, in Region 3, Tarlac State University is the Top SUC with an overall rating of 8.5 followed by Pampanga State Agricultural University with an overall rating of 8.4.

In the first batch of the result, it is visible that the University of the Philippines garnered the highest points with an overall rating of 8.9. This proves that the University is not only top in the most number of Center of Excellence and Center of Development ${ }^{12}$, but also the model SUC website in the country.This shows that the University of the Philippines is excellent not just in academic but also in web rankings.

Laguna State Polytechnic University, formerly known as Laguna State Polytechnic College was the first in the Region 4-A, and Occidental Mindoro State College was the first in Region 4-B with both rating of 8.2. Sorsogon State College was the top in Region 5 with an overall rating of 8.0. Capiz State University was the top in the Region 6 with an overall rating of 8.6. In Region 7, Cebu Technological University with an overall rating of 8.6 was the highest in the region. For the second batch of results, both Cebu Technological University and Capiz State University tied with an overall rating of 8.6.

Based on Figure 1, Majority of the website are marked as Good with a total of 70 websites, 11 of the SUC has no website, offline or using .gov.ph domain. While 23 of the SUC website has an excellent rating, there are only 10 of the SUC website are marked as Poor. This shows that SUC in the Philippines are not left behind in providing information from the institution where school websites are providing education settings with the opportunity to transform and enhance the schooling experience ${ }^{13}$.

While it was noticed that some SUC website has been not updated for some time, it is recommended that the 


\begin{tabular}{|c|c|c|c|c|c|c|c|c|c|c|c|c|c|c|c|c|c|c|c|c|c|c|c|c|c|c|c|}
\hline ТTУУG $\Lambda$ O & $\stackrel{\infty}{\sim}$ & $\stackrel{n}{n}$ & $\begin{array}{l}0 \\
\infty\end{array}$ & تే & $\stackrel{m}{+}$ & $\stackrel{n}{2}$ & ${ }_{\infty}^{+1}$ & 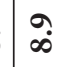 & $\grave{n}$ & 9 & 年 & $\begin{array}{l}\infty \\
1 \\
i\end{array}$ & $\begin{array}{c}m \\
\infty\end{array}$ & $\stackrel{m}{+}$ & $\stackrel{b}{\circ}$ & & సֶ. & $\bar{\infty}$ & \begin{tabular}{ll|} 
\\
\end{tabular} & \begin{tabular}{l|}
7 \\
$\infty$
\end{tabular} & $\overrightarrow{i n}$ & $\stackrel{?}{n}$ & $\stackrel{\Upsilon}{n}$ & $\stackrel{\infty}{n}$ & $\stackrel{\Upsilon}{N}$ & ִִי & فे \\
\hline lew.sof TY & $\stackrel{\circ}{+}$ & $\begin{array}{l}0 \\
\dot{0}\end{array}$ & $\begin{array}{l}0 \\
0 \\
0\end{array}$ & $\stackrel{0}{\stackrel{0}{+}}$ & $\begin{array}{l}0 \\
0\end{array}$ & $\begin{array}{l}0 \\
\dot{r}\end{array}$ & $\stackrel{\circ}{\circ}$ & $\stackrel{\circ}{\circ}$ & $\stackrel{\circ}{\circ}$ & $\stackrel{0}{0}$ & $\begin{array}{l}0 \\
0 \\
0\end{array}$ & $\underset{\infty}{+1}$ & $\begin{array}{l}0 \\
\dot{0}\end{array}$ & $\begin{array}{l}0 \\
0\end{array}$ & $\stackrel{\circ}{\therefore}$ & & $\begin{array}{l}0 \\
\stackrel{0}{0}\end{array}$ & $\stackrel{0}{\circ}$ & $\begin{array}{l}0 \\
\dot{0}\end{array}$ & $\begin{array}{l}\infty \\
\infty \\
\infty\end{array}$ & $\begin{array}{l}0 \\
0 \\
0\end{array}$ & $\begin{array}{l}0 \\
0 \\
0\end{array}$ & $\begin{array}{l}\infty \\
\infty \\
\infty\end{array}$ & $\stackrel{0}{+}$ & 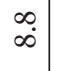 & $\begin{array}{l}\stackrel{0}{0} \\
\stackrel{0}{1}\end{array}$ & $\infty$ \\
\hline IวH!ML & & ' & ' & ' & . & 1 & $\stackrel{\circ}{\circ}$ & $\because$ & 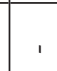 & in & ' & ' & ' & ' & & ' & ' & ' & I & ' & ' & & & ' & ' & $\stackrel{\infty}{\wedge}$ & \\
\hline 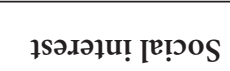 & $\stackrel{m}{n}$ & $\hat{n}$ & $\stackrel{0}{\circ}$ & $\stackrel{b}{i}$ & $\vec{n}$ & $\begin{array}{l}0 \\
\infty\end{array}$ & $\stackrel{\infty}{n}$ & 9 & $\stackrel{0}{\circ}$ & $\vec{r}$ & $\stackrel{n}{n}$ & . & $n$ & $\begin{array}{l}7 \\
0\end{array}$ & $\stackrel{0}{6}$ & & 1 & $\stackrel{\circ}{\wedge}$ & $\begin{array}{l}0 \\
0\end{array}$ & $\tilde{b}$ & ' & $\stackrel{m}{n}$ & $\stackrel{n}{2}$ & $\stackrel{m}{n}$ & ' & $\begin{array}{c}\infty \\
i \\
i\end{array}$ & $\stackrel{\circ}{i}$ \\
\hline 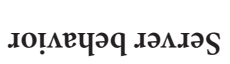 & $\stackrel{\circ}{+}$ & $\begin{array}{l}0 \\
0 \\
0\end{array}$ & $\stackrel{m}{i}$ & $\stackrel{\sharp}{6}$ & $\stackrel{0}{+}$ & $\begin{array}{l}0 \\
\dot{0}\end{array}$ & $\stackrel{0}{r}$ & $\because$ & $\stackrel{0}{m}$ & $\ddot{H}$ & $\begin{array}{ll}n \\
\text { in }\end{array}$ & $\begin{array}{l}0 \\
i\end{array}$ & 0 & $\tilde{n}$ & $\underset{\infty}{\infty}$ & & $\stackrel{6}{6}$ & $\stackrel{0}{\Lambda}$ & $\stackrel{9}{n}$ & $\begin{array}{l}0 \\
0 \\
0\end{array}$ & in & $\stackrel{\leftrightarrow}{+}$ & $\stackrel{0}{0}$ & $\vec{a}$ & $\underset{0}{0}$ & $\stackrel{0}{\circ}$ & \\
\hline 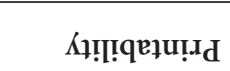 & $\because$ & $\mid \begin{array}{l}0 \\
\dot{0}\end{array}$ & $\because$ & $\stackrel{0}{0}$ & ' & ' & $\stackrel{\circ}{\circ}$ & $\because$ & $\because$ & ir & ' & ' & $\stackrel{0}{0}$ & ' & $\stackrel{0}{0}$ & & 1 & $\stackrel{0}{0}$ & $\begin{array}{l}0 \\
\dot{0} \\
\end{array}$ & $\begin{array}{l}0 \\
\dot{0}\end{array}$ & , & $\because$ & ' & $\ddot{0}$ & $\stackrel{\dot{0}}{0}$ & $\stackrel{0}{0}$ & \\
\hline 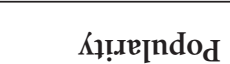 & $\curvearrowright$ & $\begin{array}{l}\ddot{H} \\
\dot{n}\end{array}$ & $\because$ & $\stackrel{0}{\wedge}$ & $\begin{array}{l}0 \\
1\end{array}$ & $\stackrel{+}{\Lambda}$ & $\stackrel{0}{1}$ & $\stackrel{n}{n}$ & $\ddot{0}$ & $\stackrel{9}{+}$ & $\underset{+}{q}$ & $\stackrel{+}{\mp}$ & $\hat{\vartheta}$ & 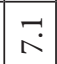 & $\stackrel{\leftrightarrow}{i}$ & & $\widehat{\emptyset}$ & $\hat{\sigma}$ & $\mid \begin{array}{l}\infty \\
i n \\
i n\end{array}$ & $\stackrel{\odot}{+}$ & $\mid \begin{array}{l}\infty \\
i\end{array}$ & ตे & $\overrightarrow{6}$ & $\stackrel{\leftrightarrow}{*}$ & $\hat{m}$ & 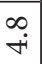 & ชู \\
\hline 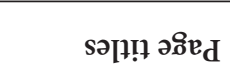 & $\stackrel{0}{0}$ & $\begin{array}{l}0 \\
\dot{0}\end{array}$ & $\begin{array}{l}0 \\
\stackrel{-}{0}\end{array}$ & $\stackrel{\circ}{\circ}$ & , & $\stackrel{0}{\circ}$ & $\stackrel{\circ}{0}$ & $\stackrel{\circ}{\circ}$ & $\stackrel{\circ}{\circ}$ & O̊. & $\stackrel{\circ}{\circ}$ & $\stackrel{\circ}{\circ}$ & $\begin{array}{l}0 \\
\dot{0}\end{array}$ & , & $\stackrel{\circ}{\circ}$ & & $\begin{array}{l}0 \\
\stackrel{0}{-}\end{array}$ & $\begin{array}{l}0 \\
0\end{array}$ & $\begin{array}{l}\dot{0} \\
\dot{0}\end{array}$ & 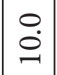 & ir & $\begin{array}{l}0 \\
\stackrel{0}{0}\end{array}$ & $\begin{array}{l}\stackrel{0}{0} \\
\stackrel{0}{1}\end{array}$ & $\begin{array}{l}0 \\
\stackrel{0}{0}\end{array}$ & $\begin{array}{l}0 \\
\stackrel{0}{0}\end{array}$ & $\begin{array}{l}\stackrel{0}{\circ} \\
\stackrel{0}{1}\end{array}$ & $\stackrel{\circ}{\circ}$ \\
\hline әा!q०М & $\stackrel{0}{0}$ & $\begin{array}{l}0 \\
0 \\
0\end{array}$ & $\stackrel{0}{0}$ & $\stackrel{0}{\dot{0}}$ & $\dot{m}$ & $\begin{array}{l}0 \\
\dot{0}\end{array}$ & $\stackrel{\circ}{\circ}$ & $\stackrel{\circ}{\circ}$ & $\stackrel{\circ}{\circ}$ & $\stackrel{0}{0}$ & $\begin{array}{l}0 \\
\dot{0}\end{array}$ & $\stackrel{0}{\infty}$ & $\begin{array}{l}0 \\
0 \\
0\end{array}$ & $\ddot{r}$ & $\stackrel{\circ}{\circ}$ & & $\stackrel{\circ}{\dot{r}}$ & $\begin{array}{l}0 \\
\dot{0}\end{array}$ & $\begin{array}{l}0 \\
\dot{0} \\
\end{array}$ & $\begin{array}{l}0 \\
\dot{0}\end{array}$ & $\begin{array}{l}0 \\
\stackrel{0}{0}\end{array}$ & $\begin{array}{l}0 \\
\dot{0}\end{array}$ & $\begin{array}{l}\stackrel{0}{0} \\
\stackrel{0}{1}\end{array}$ & $\begin{array}{l}0 \\
\stackrel{0}{0}\end{array}$ & $\begin{array}{l}0 \\
\infty \\
\infty\end{array}$ & ஸี & $\begin{array}{l}\stackrel{0}{0} \\
\stackrel{0}{0}\end{array}$ \\
\hline 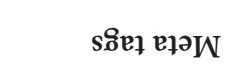 & $\stackrel{\circ}{\circ}$ & $\stackrel{0}{i}$ & $\stackrel{\circ}{i}$ & $\stackrel{\circ}{i}$ & , & $\stackrel{\circ}{i}$ & $\stackrel{0}{0}$ & $\because$ & $\stackrel{\circ}{i}$ & $\dot{0}$ & $\stackrel{0}{i}$ & $\stackrel{\circ}{i}$ & $\stackrel{0}{i}$ & $\stackrel{0}{i}$ & $\stackrel{\circ}{i}$ & & $\stackrel{\circ}{i}$ & $\stackrel{r}{\dot{n}}$ & $\stackrel{i}{i}$ & $\because$ & $\stackrel{o}{i}$ & $\stackrel{\circ}{i}$ & $\stackrel{0}{\circ}$ & $\stackrel{\circ}{i}$ & $\stackrel{0}{\circ}$ & $\stackrel{\circ}{i}$ & $\stackrel{\circ}{\circ}$ \\
\hline syu!̣ Ir & $\stackrel{0}{\circ}$ & 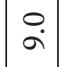 & $\ddot{a}$ & $\stackrel{\infty}{\infty}$ & $\dot{0}$ & $\dot{0}$ & $\stackrel{0}{\circ}$ & F & $\approx$ & $\stackrel{0}{\circ}$ & \begin{tabular}{|l|}
10 \\
$\infty$
\end{tabular} & $\hat{a}$ & $\left|\begin{array}{l}0 \\
\infty\end{array}\right|$ & $\begin{array}{l}0 \\
0 \\
\end{array}$ & $\stackrel{\circ}{\circ}$ & & $\stackrel{\infty}{0}$ & 움 & 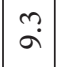 & $\begin{array}{l}0 \\
0 \\
0\end{array}$ & $\because$ & $\stackrel{N}{N}$ & $\hat{\sigma}$ & กั & $\begin{array}{l}+1 \\
\infty\end{array}$ & $\begin{array}{l}0 \\
\bullet \\
0\end{array}$ & $\underbrace{0}_{0}$ \\
\hline 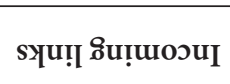 & $\stackrel{0}{\infty}$ & $\underset{+}{\stackrel{P}{+}}$ & $\begin{array}{l}0 \\
0 \\
0\end{array}$ & in & $\begin{array}{l}0 \\
\dot{0}\end{array}$ & $\begin{array}{l}0 \\
0 \\
0\end{array}$ & $\stackrel{0}{0}$ & $\stackrel{\circ}{\ominus}$ & $\stackrel{0}{0}$ & $\stackrel{0}{0}$ & $\begin{array}{l}0 \\
\dot{9}\end{array}$ & $\stackrel{\infty}{-}$ & $\begin{array}{l}0 \\
0 \\
0\end{array}$ & $\begin{array}{l}0 \\
0\end{array}$ & $\begin{array}{l}\infty \\
0 \\
0\end{array}$ & ' & $\overrightarrow{0}$ & $\begin{array}{l}0 \\
\dot{0}\end{array}$ & $\stackrel{\infty}{-i}$ & 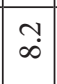 & ' & $\begin{array}{l}0 \\
\dot{0}\end{array}$ & $\vec{n}$ & $\begin{array}{l}0 \\
\dot{0}\end{array}$ & $\begin{array}{l}0 \\
\stackrel{0}{0}\end{array}$ & $\begin{array}{l}\stackrel{0}{0} \\
\stackrel{9}{1}\end{array}$ & ชู \\
\hline səร̊cuा & $\vec{\infty}$ & \begin{tabular}{|l|}
10 \\
$\infty$
\end{tabular} & $\vec{a}$ & $\begin{array}{l}0 \\
\infty\end{array}$ & $\begin{array}{l}0 \\
0 \\
0\end{array}$ & $\ddot{a}$ & $\because$ & $\stackrel{n}{\infty}$ & N & $\stackrel{0}{0}$ & $\begin{array}{l}10 \\
\alpha\end{array}$ & $\stackrel{\sim}{i}$ & $\infty$ & $\begin{array}{l}0 \\
0\end{array}$ & $\stackrel{0}{\infty}$ & & 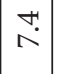 & $\infty$ & 2 & $\begin{array}{l}\hat{\infty} \\
\infty\end{array}$ & $\stackrel{0}{0}$ & $\hat{a}$ & $\ddot{a}$ & $\stackrel{\infty}{a}$ & $\tilde{a}$ & $\hat{\sigma}$ & $\ddot{\sigma}$ \\
\hline 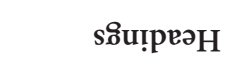 & 2 & $\stackrel{\circ}{9}$ & $\ddot{a}$ & q & , & $\circ$ & $\stackrel{r}{f}$ & $\stackrel{.}{\circ}$ & $\because$ & $\ddot{0}$ & $\check{a}$ & $\stackrel{0}{\Lambda}$ & $\left|\begin{array}{l}0 \\
\infty\end{array}\right|$ & , & in & , & $\stackrel{0}{0}$ & $\begin{array}{c}a \\
\infty\end{array} \mid$ & 우 & $\ddot{0}$ & , & :ं & in. & $\stackrel{\leftrightarrow}{+}$ & $\hat{\sigma}$ & $\stackrel{0}{\circ}$ & $\infty_{\infty}$ \\
\hline ssəuપsə.ાH & $\stackrel{\circ}{\circ}$ & \begin{tabular}{|l|} 
\\
$\dot{9}$
\end{tabular} & $\begin{array}{l}0 \\
0 \\
0\end{array}$ & $\begin{array}{l}0 \\
\dot{0}\end{array}$ & $\stackrel{\vec{i}}{\mathrm{i}}$ & $\begin{array}{l}0 \\
\dot{9}\end{array}$ & $\stackrel{\circ}{\circ}$ & $\stackrel{\circ}{\circ}$ & $\stackrel{\circ}{\circ}$ & $\stackrel{\circ}{\circ}$ & $\stackrel{0}{0}$ & $\stackrel{\circ}{0}$ & $\begin{array}{l}0 \\
0 \\
0\end{array}$ & -7 & $\stackrel{0}{\circ}$ & & $\begin{array}{l}0 \\
0 \\
0\end{array}$ & $\begin{array}{l}0 \\
\dot{0} \\
\end{array}$ & $\stackrel{\circ}{\circ}$ & $\begin{array}{l}\dot{0} \\
\dot{0}\end{array}$ & $\begin{array}{l}0 \\
\dot{0}\end{array}$ & $\begin{array}{l}0 \\
\stackrel{0}{0}\end{array}$ & $\stackrel{\circ}{\stackrel{\bullet}{0}}$ & $\begin{array}{l}0 \\
\stackrel{0}{-}\end{array}$ & $\begin{array}{l}\dot{0} \\
\stackrel{0}{0}\end{array}$ & $\begin{array}{l}0 \\
\dot{0}\end{array}$ & $\stackrel{\circ}{\circ}$ \\
\hline 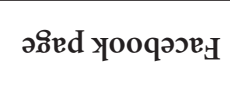 & $a$ & ' & ' & ' & ' & $\begin{array}{l}0 \\
\dot{0}\end{array}$ & $\stackrel{\circ}{\circ}$ & $\stackrel{\circ}{\stackrel{9}{9}}$ & ' & $\stackrel{\wedge}{\infty}$ & $\ddot{0}$ & $\begin{array}{l}1 \\
\infty \\
\infty\end{array}$ & $\stackrel{0}{0}$ & ' & & ' & ' & $\ddot{a}$ & $\left|\begin{array}{l}1 \\
\infty \\
\infty\end{array}\right|$ & $\begin{array}{l}a \\
\infty\end{array}$ & ' & $\begin{array}{l}\infty \\
\infty \\
\infty\end{array}$ & $\stackrel{?}{?}$ & $\stackrel{0}{0}$ & , & $\stackrel{\circ}{\circ}$ & ' \\
\hline sọฺK[euV & . & ' & $\stackrel{0}{0}$ & , & , & ' & , & $\stackrel{\circ}{\stackrel{\leftrightarrow}{9}}$ & $\stackrel{\circ}{\circ}$ & ' & $\begin{array}{l}0 \\
\stackrel{\leftrightarrow}{0}\end{array}$ & $\stackrel{\circ}{+}$ & $\stackrel{0}{0}$ & , & $\stackrel{\circ}{\circ}$ & & $\begin{array}{l}0 \\
\stackrel{0}{0}\end{array}$ & O̊. & . & $\stackrel{\circ}{\circ}$ & ' & . & , & $\stackrel{\circ}{\circ}$ & ' & ' & ' \\
\hline $\begin{array}{r}\text { ұuəłuos } \\
\text { jo ұunour }\end{array}$ & $\stackrel{\infty}{\wedge}$ & $\stackrel{n}{n}$ & $\stackrel{0}{\infty}$ & $\begin{array}{l}a \\
\infty\end{array}$ & , & $\stackrel{\circ}{\Lambda}$ & an & 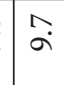 & $\begin{array}{l}m \\
\infty \\
\infty\end{array}$ & $\stackrel{0}{0}$ & $\begin{array}{l}0 \\
0 \\
\infty\end{array}$ & $\begin{array}{l}n \\
\infty\end{array}$ & $\stackrel{0}{1}$ & , & กุ & , & 苗 & $\because$ & $\mid \begin{array}{l}n \\
\infty\end{array}$ & \begin{tabular}{|l|}
$n$ \\
$\infty$
\end{tabular} & $\ddot{6}$ & $\begin{array}{l}n \\
\infty \\
\infty\end{array}$ & $\stackrel{0}{\circ}$ & $\tilde{n}$ & $\begin{array}{l}\infty \\
\infty\end{array}$ & $\stackrel{n}{\infty}$ & $\begin{array}{l}0 \\
\infty\end{array}$ \\
\hline uo!̣̊ว & $\begin{array}{l}\text { 년 } \\
\text { Z }\end{array}$ & \begin{tabular}{l|}
\multicolumn{1}{|c}{} \\
$\mathbf{U}$ \\
$Z$
\end{tabular} & 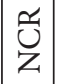 & 总 & $\begin{array}{l}\approx \\
0 \\
z\end{array}$ & $\begin{array}{l}\text { 寽 } \\
z\end{array}$ & 总 & $\begin{array}{l}\text { 妾 } \\
z\end{array}$ & - & - & $\neg$ & $\neg$ & - & -1 & 竎 & 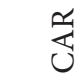 & 离 & 胥 & 色 & 㞼 & $\sim$ & $N$ & $\mathrm{~N}$ & $\sim$ & $\sim$ & $m$ & $m$ \\
\hline 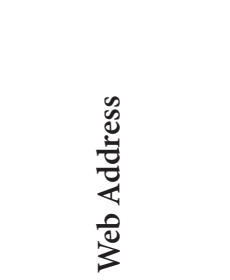 & 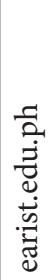 & 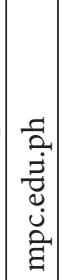 & 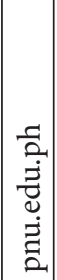 & 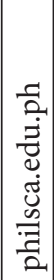 & 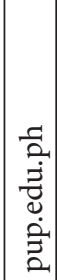 & 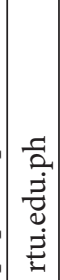 & 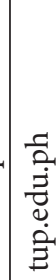 & 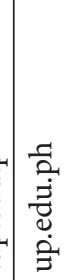 & 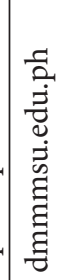 & 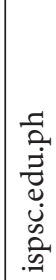 & 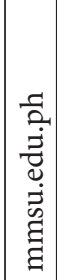 & 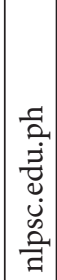 & 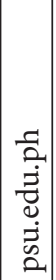 & 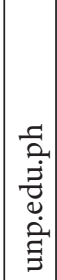 & 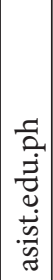 & 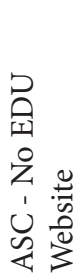 & 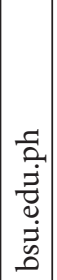 & 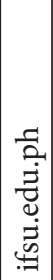 & 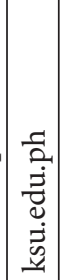 & 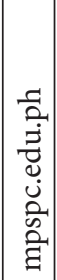 & 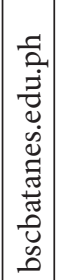 & 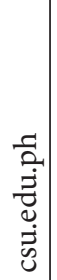 & 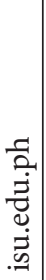 & 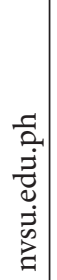 & 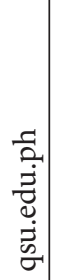 & 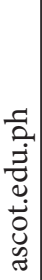 & 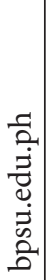 \\
\hline
\end{tabular}




\begin{tabular}{|c|c|c|c|c|c|c|c|c|c|c|}
\hline ТТУХА & $\stackrel{ }{N}$ & $\dddot{n}$ & in & $\tilde{0}$ & $\underset{\infty}{\sim}$ & $\underset{\infty}{+\infty}$ & بִ & $\stackrel{+}{\top}$ & $\begin{array}{l}0 \\
\end{array}$ & 1n \\
\hline 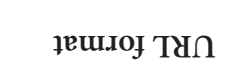 & $\begin{array}{l}\infty \\
\infty\end{array}$ & $\stackrel{\circ}{0}$ & in & $\begin{array}{l}0 \\
\dot{n}\end{array}$ & $\begin{array}{l}0 \\
0 \\
1\end{array}$ & $\begin{array}{l}\infty \\
\infty\end{array}$ & $\begin{array}{l}\infty \\
\infty \\
\infty\end{array}$ & $\stackrel{0}{0}$ & $\stackrel{0}{0}$ & $\stackrel{\infty}{\infty}$ \\
\hline .Јગ!!ML & ' & I & ' & ' & ' & $\ddot{H}$ & ' & ' & ' &. \\
\hline 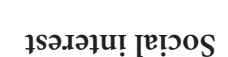 & $\begin{array}{l}0 \\
\dot{0}\end{array}$ & $\begin{array}{l}0 \\
\end{array}$ & $\stackrel{\circ}{\circ}$ & $\underset{0}{\infty}$ & $\stackrel{?}{\sim}$ & 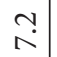 & $\stackrel{\infty}{\sim}$ & 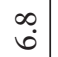 & $\stackrel{N}{N}$ & $\stackrel{n}{n}$ \\
\hline 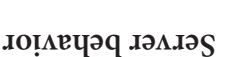 & $\begin{array}{l}\sigma \\
\ddot{0}\end{array}$ & $\stackrel{\circ}{\circ}$ & $\ddot{H}$ & مبْ & $\stackrel{\infty}{\sim}$ & $\stackrel{\varphi}{\ddot{r}}$ & $\stackrel{9}{m}$ & $\stackrel{0}{\circ}$ & $\underset{\infty}{\infty}$ & சै. \\
\hline র!!!!qęu!...d & $\stackrel{0}{0}$ & $\stackrel{\circ}{0}$ & $\stackrel{\circ}{\circ}$ & ' & $\begin{array}{l}0 \\
\infty\end{array}$ & $\stackrel{0}{0}$ & $\begin{array}{l}0 \\
\infty\end{array}$ & $\stackrel{\circ}{\circ}$ & $\stackrel{0}{0}$ & $\stackrel{0}{0}$ \\
\hline 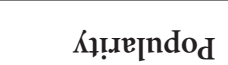 & $\stackrel{m}{\not}$ & ֶֶ? & $\widehat{\vartheta}$ & $\begin{array}{l}n \\
\text { in }\end{array}$ & $\stackrel{\wedge}{i n}$ & ○े & $\stackrel{n}{n}$ & $\vec{n}$ & \begin{tabular}{l}
0 \\
\hdashline
\end{tabular} & $\begin{array}{l}0 \\
0\end{array}$ \\
\hline 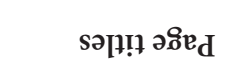 & $\stackrel{\circ}{\circ}$ & $\stackrel{\circ}{\circ}$ & $\stackrel{\circ}{\circ}$ & $\underset{\infty}{\infty}$ & $\stackrel{0}{0}$ & $\stackrel{0}{\circ}$ & $\stackrel{\circ}{\circ}$ & $\stackrel{\circ}{\circ}$ & $\stackrel{\circ}{0}$ & $\stackrel{0}{0}$ \\
\hline ә!ฺоW & $\stackrel{\circ}{\circ}$ & $\stackrel{\circ}{\circ}$ & $\stackrel{0}{\circ}$ & $\begin{array}{l}0 \\
\infty\end{array}$ & $\begin{array}{l}0 \\
\infty\end{array}$ & $\stackrel{0}{0}$ & $\begin{array}{l}0 \\
\infty\end{array}$ & $\stackrel{\circ}{\circ}$ & $\stackrel{0}{\circ}$ & 웅 \\
\hline s.̊ำ еาวW & $\stackrel{\circ}{\circ}$ & $\stackrel{\circ}{i}$ & $\stackrel{\circ}{i}$ & $\underset{\infty}{\infty}$ & $\underset{\infty}{+1}$ & $\stackrel{\circ}{\circ}$ & $\stackrel{\circ}{\mathrm{i}}$ & $\stackrel{\circ}{\mathrm{i}}$ & $\stackrel{0}{0}$ & $\stackrel{\circ}{i}$ \\
\hline 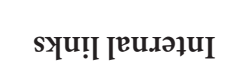 & 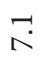 & $\begin{array}{l}+! \\
\infty\end{array}$ & $\stackrel{\sim}{\sim}$ & $\stackrel{\sigma}{\infty}$ & $\underset{\infty}{\infty}$ & $\stackrel{\circ}{\circ}$ & $\stackrel{\circ}{\circ}$ & $\stackrel{\varrho}{\infty}$ & nְ. & $\ddot{\sigma}$ \\
\hline syụ̣ ̣̣̊̊uosuI & $\stackrel{\curvearrowright}{\curvearrowright}$ & $\stackrel{\circ}{\circ}$ & $\stackrel{0}{0}$ & $\stackrel{0}{\circ}$ & $\stackrel{0}{0}$ & $\stackrel{\circ}{\circ}$ & $\stackrel{0}{0}$ & $\stackrel{0}{0}$ & $\begin{array}{l}0 \\
0\end{array}$ & O. \\
\hline sวริeนI & $\begin{array}{l}10 \\
\infty\end{array}$ & $\begin{array}{l}0 \\
0 \\
0\end{array}$ & $\stackrel{\curvearrowright}{\infty}$ & ๙̃ & $\stackrel{\infty}{\sigma}$ & $\tilde{n}$ & $\tilde{a}$ & $\stackrel{\circ}{\circ}$ & aे & ๙ூ \\
\hline sôu!peəH & $\stackrel{0}{\sim}$ & $\begin{array}{l}0 \\
\text { in }\end{array}$ & 아. & $\stackrel{0}{\dot{m}}$ & $\stackrel{0}{\infty}$ & $\stackrel{\circ}{\circ}$ & $\stackrel{\circ}{1}$ & $\stackrel{n}{n}$ & $\begin{array}{l}0 \\
0\end{array}$ & $\underline{a}$ \\
\hline ssəપપડ્.ાH & $\stackrel{0}{0}$ & $\stackrel{\circ}{0}$ & $\stackrel{0}{0}$ & $\stackrel{\circ}{\circ}$ & $\stackrel{0}{0}$ & $\stackrel{\circ}{\circ}$ & $\stackrel{\circ}{\circ}$ & $\stackrel{\circ}{0}$ & $\stackrel{0}{0}$ & 웅 \\
\hline วภ̊ed уооорәәе & 1 & ' & ' & $\begin{array}{l}0 \\
0\end{array}$ & , & $\begin{array}{l}0 \\
0\end{array}$ & , & ' & , & 1 \\
\hline 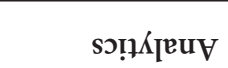 & ' & $\stackrel{\circ}{i}$ & ' & ' & $\underset{\infty}{0}$ & ' & $\stackrel{\circ}{\infty}$ & , & , & $\stackrel{\circ}{\circ}$ \\
\hline 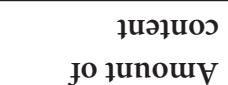 & $\stackrel{\infty}{\infty}$ & $\underset{\infty}{-1}$ & $\stackrel{n}{n}$ & $\stackrel{N}{N}$ & ạ & $\ddot{a}$ & $\stackrel{0}{\circ}$ & $\stackrel{1}{\infty}$ & $\begin{array}{l}0 \\
\infty\end{array}$ & $\stackrel{9}{1}$ \\
\hline บo!̣ภวิy & $m$ & $m$ & $m$ & $m$ & $m$ & $m$ & $m$ & $m$ & $m$ & $m$ \\
\hline 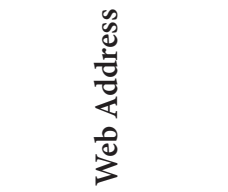 & 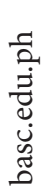 & 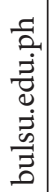 & 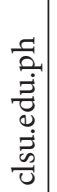 & 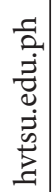 & 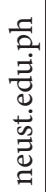 & 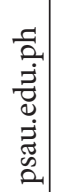 & 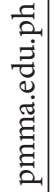 & 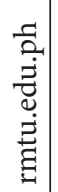 & 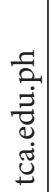 & 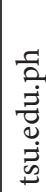 \\
\hline
\end{tabular}

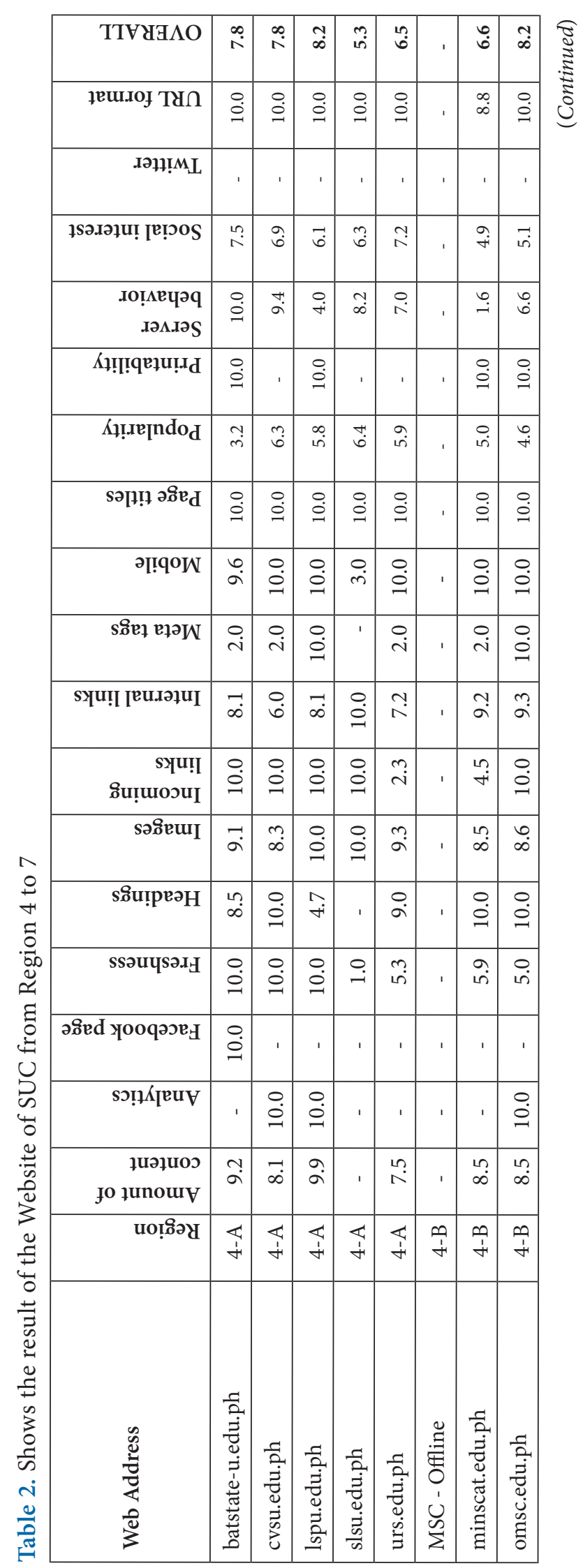




\begin{tabular}{|c|c|c|c|c|c|c|c|c|c|c|c|c|c|c|c|c|c|c|c|c|c|c|c|}
\hline ТТУ४А $\Lambda$ & $\stackrel{+}{\stackrel{4}{n}}$ & $\vec{n}$ & in & $\underset{+}{0}$ & $\stackrel{1 ?}{?}$ & $\begin{array}{l}\infty \\
\infty \\
\dot{0}\end{array}$ & $\stackrel{\square}{\therefore}$ & : & $\begin{array}{l}\infty \\
\stackrel{0}{0}\end{array}$ & $\stackrel{\Upsilon}{\Re}$ & 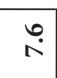 & $\stackrel{\circ}{\infty}$ & $\begin{array}{l}\stackrel{0}{0} \\
\dot{0}\end{array}$ & $\begin{array}{l}0 \\
\infty \\
\infty\end{array}$ & $\stackrel{\circ}{i}$ & $\stackrel{\Upsilon}{\wedge}$ & 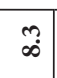 & $\hat{6}$ & $\stackrel{+}{i}$ & $\stackrel{19}{1}$ & 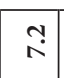 & $m$ & $\stackrel{\imath}{\wedge}$ \\
\hline деш.оу ТУด & $\begin{array}{l}\infty \\
\infty\end{array}$ & $\stackrel{\circ}{\circ}$ & $\stackrel{\circ}{\circ}$ & $\stackrel{\circ}{\circ}$ & $\stackrel{\circ}{\Theta}$ & $\stackrel{\circ}{\dot{m}}$ & $\stackrel{\circ}{\circ}$ & $\stackrel{\circ}{\dot{m}}$ & $\stackrel{\circ}{\circ}$ & $\begin{array}{l}\infty \\
\infty \\
\infty\end{array}$ & $\begin{array}{l}\infty \\
\infty \\
\infty\end{array}$ & $\stackrel{\circ}{\stackrel{-}{0}}$ & $\begin{array}{l}\infty \\
\infty \\
\infty\end{array}$ & $\begin{array}{l}\infty \\
\infty\end{array}$ & $\begin{array}{l}\infty \\
\infty\end{array}$ & $\stackrel{0}{\dot{\Theta}}$ & $\stackrel{\circ}{\stackrel{9}{9}}$ & $\stackrel{\circ}{\circ}$ & $\begin{array}{l}\infty \\
\infty \\
\infty\end{array}$ & $\stackrel{\circ}{\circ}$ & $\stackrel{\circ}{\circ}$ & $\stackrel{\circ}{\stackrel{0}{0}}$ & $\stackrel{\circ}{\stackrel{9}{9}}$ \\
\hline. & & & & & & & & & & & $\stackrel{\infty}{\dot{m}}$ & $\stackrel{m}{+}$ & & & $\overrightarrow{6}$ & & & & & & & & \\
\hline 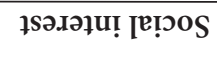 & ఫે & ?ై & in & $\stackrel{\circ}{\therefore}$ & $\stackrel{0}{\circ}$ & $\stackrel{n}{6}$ & 甪 & రి & ?ु. & 9 & $\underset{F}{\mathcal{F}}$ & $\stackrel{\circ}{\wedge}$ & $\underset{+}{\vec{f}}$ & $\stackrel{\infty}{0}^{\infty}$ & 8 & $\vec{\pi}$ & $\stackrel{+}{i}$ & $\stackrel{\infty}{0}^{\circ}$ & $\stackrel{\circ}{\dot{m}}$ & $\begin{array}{l}0 \\
b\end{array}$ & $\stackrel{\imath}{i n}$ & $\stackrel{⿱ 亠 幺}{N}$ & $\frac{\sqrt{3}}{3}$ \\
\hline 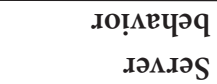 & $\stackrel{2}{\Omega}$ & $\stackrel{\circ}{9}$ & $\underset{\infty}{\infty}$ & $\underset{\infty}{\infty}$ & $\stackrel{\circ}{\circ}$ & $\stackrel{\circ}{\Theta}$ & $\stackrel{\circ}{0}$ & $\underset{+}{0}$ & : & $\begin{array}{ll}0 \\
+ \\
+\end{array}$ & $\stackrel{\bullet}{\dot{m}}$ & $\stackrel{\circ}{\circ}$ & $\overbrace{0}^{0}$ & $\stackrel{+}{+}$ & $\stackrel{+}{n}$ & $\stackrel{\circ}{\varrho}$ & $\stackrel{\circ}{\stackrel{9}{9}}$ & ชู & రి & tit & $\underset{+}{0}$ & $\stackrel{\circ}{\stackrel{0}{0}}$ & $\stackrel{\circ}{\circ}$ \\
\hline ห!!! & $\stackrel{\circ}{0}$ & ' & ' & ' & $\stackrel{0}{0}$ & $\stackrel{\circ}{\stackrel{0}{0}}$ & $\stackrel{0}{0}$ & $\stackrel{0}{0}$ & $\stackrel{\circ}{0}$ & $\stackrel{0}{0}$ & $\stackrel{0}{0}$ & $\stackrel{\circ}{0}$ & ' & $\stackrel{0}{0}$ & & $\stackrel{\circ}{\circ}$ & $\stackrel{\circ}{\circ}$ & $\stackrel{\circ}{\circ}$ & $\stackrel{0}{\dot{\Theta}}$ & ' & $\stackrel{0}{\dot{0}}$ & $\stackrel{\circ}{\circ}$ & $\stackrel{\circ}{\circ}$ \\
\hline หৃ!.xp[ndo & in & $\stackrel{?}{?}$ & in & $\stackrel{g}{r}$ & $\hat{\dot{m}}$ & $\stackrel{L}{+}$ & ir & in & $\stackrel{?}{n}$ & $\stackrel{m}{+}$ & 年 & $\stackrel{\infty}{\infty}$ & $\stackrel{\infty}{+}$ & $\stackrel{\leftrightarrow}{-}$ & $\stackrel{\infty}{i}$ & $\exists$ & $\curvearrowright$ & $\begin{array}{l}n \\
+ \\
+\end{array}$ & $\stackrel{m}{m}$ & $\vec{m}$ & $\stackrel{m}{n}$ & $\stackrel{\curvearrowright}{\wedge}$ & $\stackrel{\circ}{i}$ \\
\hline 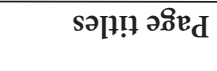 & $\stackrel{\circ}{\stackrel{0}{-}}$ & $\stackrel{\circ}{\circ}$ & $\stackrel{\circ}{\dot{\theta}}$ & & $\stackrel{\circ}{\stackrel{\theta}{0}}$ & $\stackrel{\circ}{\Theta}$ & $\stackrel{0}{\dot{9}}$ & $\stackrel{0}{\stackrel{\Theta}{-}}$ & $\stackrel{\circ}{\Theta}$ & $\stackrel{0}{\stackrel{\oplus}{\oplus}}$ & $\stackrel{0}{\stackrel{9}{9}}$ & $\stackrel{\circ}{0}$ & $\stackrel{\circ}{\stackrel{0}{0}}$ & $\stackrel{\circ}{\stackrel{0}{0}}$ & $\stackrel{\circ}{\circ}$ & $\stackrel{\circ}{\circ}$ & $\stackrel{\circ}{\stackrel{-}{0}}$ & $\stackrel{\circ}{9}$ & $\stackrel{\circ}{\stackrel{9}{0}}$ & $\stackrel{\circ}{\stackrel{\dot{9}}{1}}$ & $\begin{array}{l}\dot{9} \\
\dot{\theta}\end{array}$ & $\stackrel{\circ}{9}$ & $\stackrel{\circ}{\circ}$ \\
\hline ә!!१०Џ & $\stackrel{?}{\Upsilon}$ & $\stackrel{\circ}{\circ}$ & $\ddot{m}$ & $\stackrel{\circ}{\dot{m}}$ & $\stackrel{0}{\circ}$ & $\stackrel{\circ}{\circ}$ & $\stackrel{\circ}{\circ}$ & $\stackrel{0}{\circ}$ & $\stackrel{\circ}{\dot{m}}$ & $\stackrel{0}{\circ}$ & $\stackrel{0}{0}$ & $\stackrel{0}{\circ}$ & $\stackrel{\circ}{0}$ & $\stackrel{\circ}{\circ}$ & $\underset{\infty}{\infty}$ & $\stackrel{\circ}{\circ}$ & $\stackrel{0}{0}$ & $\stackrel{\circ}{\circ}$ & $\stackrel{\circ}{\circ}$ & $\stackrel{0}{0}$ & $\stackrel{0}{\circ}$ & $\stackrel{\circ}{\circ}$ & $\stackrel{\circ}{\circ}$ \\
\hline & $\stackrel{\circ}{\circ}$ & $\stackrel{\circ}{i}$ & $\stackrel{\circ}{i}$ & ' & $\stackrel{\circ}{i}$ & $\stackrel{\circ}{i}$ & $\begin{array}{l}\dot{r} \\
\dot{r}\end{array}$ & $\stackrel{\circ}{i}$ & $\stackrel{\circ}{i}$ & $\stackrel{0}{0}$ & $\stackrel{\circ}{i}$ & $\stackrel{\circ}{i}$ & $\stackrel{\circ}{i}$ & $\stackrel{0}{\circ}$ & $\stackrel{\circ}{i}$ & $\stackrel{\circ}{i}$ & $\begin{array}{l}\dot{0} \\
\dot{m}\end{array}$ & $\stackrel{\circ}{i}$ & $\stackrel{0}{0}$ & $\stackrel{\circ}{i}$ & $\begin{array}{l}\stackrel{i}{i} \\
\end{array}$ & $\stackrel{\leftrightarrow}{\dot{m}}$ & $\stackrel{\circ}{i}$ \\
\hline słฯ!̣! & $\underset{\infty}{\infty}$ & $\stackrel{\partial}{\infty}$ & $\stackrel{0}{\circ}$ & $\stackrel{0}{\dot{9}}$ & $\stackrel{\circ}{\circ}$ & ๙઼ & $\vec{\infty}$ & $\begin{array}{l}10 \\
\infty\end{array}$ & $\stackrel{?}{N}$ & aे & $\stackrel{\circ}{\circ}$ & $\stackrel{\vartheta}{+}$ & $\stackrel{m}{a}$ & $\vec{a}$ & $?$ & $\underset{\infty}{\infty}$ & $\stackrel{\circ}{\circ}$ & $\stackrel{\infty}{\rightarrow}$ & $\widehat{\infty}$ & $\begin{array}{l}\text { in } \\
\text { in }\end{array}$ & $\begin{array}{c}m \\
\infty\end{array}$ & $\stackrel{\circ}{\circ}$ & $\vec{\infty}$ \\
\hline $\begin{array}{r}\text { syu!I } \\
\text { 8u!̣uоэuI }\end{array}$ & $\stackrel{\circ}{\circ}$ & $\stackrel{\circ}{\circ}$ & $\ddot{\dot{9}}$ & $\begin{array}{l}0 \\
0\end{array}$ & $\frac{9}{1}$ & $\stackrel{0}{\circ}$ & $\stackrel{0}{\circ}$ & 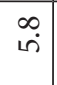 & $\stackrel{\partial}{+}$ & $\stackrel{n}{n}$ & $\stackrel{m}{i}$ & ชู & $\stackrel{\circ}{\circ}$ & $\begin{array}{l}\stackrel{0}{0} \\
\stackrel{0}{1}\end{array}$ & $\stackrel{m}{\infty}$ & $\stackrel{\circ}{\circ}$ & $\stackrel{0}{\circ}$ & $\underset{+}{F}$ & $\stackrel{n ?}{\longrightarrow}$ & $\stackrel{0}{\circ}$ & $\stackrel{0}{\circ}$ & $\stackrel{\circ}{\circ}$ & $\stackrel{\circ}{\varrho}$ \\
\hline & $\stackrel{0}{\circ}$ & กั & $\stackrel{0}{\circ}$ & $\stackrel{0}{\circ}$ & ๙ָ & $\widehat{\sigma}$ & $\stackrel{a}{\wedge}$ & $\stackrel{\circ}{\circ}$ & $\stackrel{\circ}{\circ}$ & $\stackrel{\circ}{\circ}$ & $\stackrel{0}{\circ}$ & $\stackrel{m}{a}$ & $\vec{a}$ & $\hat{\sigma}$ & 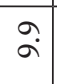 & $\vec{\infty}$ & $\stackrel{a}{\Omega}$ & $\stackrel{\circ}{\circ}$ & $\stackrel{\sim}{a}$ & $\stackrel{\circ}{\circ}$ & $\vec{\infty}$ & $\stackrel{9}{\Lambda}$ & $\vec{a}$ \\
\hline & $\stackrel{0}{0}$ & $\stackrel{\circ}{\circ}$ & ' & ' & $\stackrel{\circ}{\circ}$ & $\stackrel{0}{\circ}$ & $\stackrel{\circ}{\wedge}$ & 라 & & $\stackrel{\circ}{\circ}$ & $\stackrel{0}{\circ}$ & $\stackrel{\circ}{\circ}$ & $\stackrel{\circ}{\text { in }}$ & $\stackrel{\infty}{\sigma^{\circ}}$ & $\stackrel{\circ}{\circ}$ & $\stackrel{\circ}{\circ}$ & $\stackrel{0}{\circ}$ & 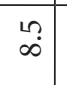 & $\stackrel{\circ}{\circ}$ & $\stackrel{\circ}{\circ}$ & $\stackrel{\infty}{\infty}$ & $\stackrel{\circ}{\circ}$ & 7 \\
\hline & $\stackrel{0}{\circ}$ & ָூ & $\stackrel{\circ}{\circ}$ & -7 & $\stackrel{\circ}{\circ}$ & $\stackrel{\circ}{\circ}$ & $\stackrel{\circ}{\circ}$ & $\stackrel{\circ}{\circ}$ & $\stackrel{\circ}{\circ}$ & $\stackrel{\circ}{\circ}$ & $\stackrel{\circ}{\circ}$ & $\stackrel{0}{\circ}$ & $\stackrel{\circ}{\circ}$ & $\stackrel{\circ}{\circ}$ & $\stackrel{\circ}{\circ}$ & $\stackrel{\sim}{\mathrm{i}}$ & $\stackrel{\circ}{\circ}$ & $\because$ & $\stackrel{\circ}{\circ}$ & $\stackrel{n}{n}$ & $\stackrel{\circ}{\circ}$ & $\stackrel{\circ}{\circ}$ & $\stackrel{0}{\circ}$ \\
\hline วक्षd yoc & & & 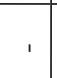 & ' & ' & ' & 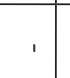 & & $\stackrel{\infty}{\wedge}$ & & ' & ' & ' & ' & : & & & $\vec{r}$ & ' & $\underset{\infty}{+}$ & , & ' & $\stackrel{\circ}{0}$ \\
\hline 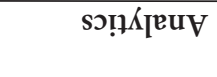 & & & & , & , & ' & & & $\stackrel{\circ}{\circ}$ & ' & $\stackrel{0}{0}$ & $\stackrel{\circ}{\circ}$ & ' & $\stackrel{\circ}{0}$ & $\stackrel{0}{0}$ & & $\stackrel{0}{0}$ & & ' & $\stackrel{\circ}{\circ}$ & ' & $\stackrel{\circ}{0}$ & \\
\hline 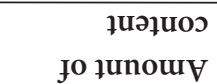 & $\stackrel{9}{\Lambda}$ & $\overrightarrow{6}$ & , & , & 1n & ?? & 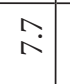 & $\begin{array}{l}\infty \\
\infty \\
\infty\end{array}$ & $\underset{\infty}{\infty}$ & $\stackrel{\infty}{\infty}$ & $\stackrel{1}{\Lambda}$ & $\begin{array}{l}1 \\
\infty \\
\infty\end{array}$ & $\underset{\infty}{0}$ & $\stackrel{\circ}{\circ}$ & $\begin{array}{l}10 \\
\infty \\
\infty\end{array}$ & $\hat{\infty}_{\infty}$ & $\begin{array}{l}0 \\
\emptyset\end{array}$ & $\vec{\infty}$ & $\stackrel{?}{\curvearrowright}$ & $\vec{\infty}$ & $a ?$ & $\overbrace{0}^{\circ}$ & $\stackrel{n}{n}$ \\
\hline uo!̣̊̊गy & $\stackrel{m}{q}$ & q & $\stackrel{m}{q}$ & in & in & in & in & in & in & in & in & in & 6 & 6 & 6 & 0 & 6 & 0 & 0 & 0 & 0 & 0 & 6 \\
\hline 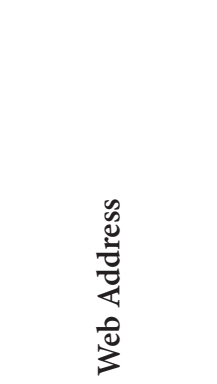 & 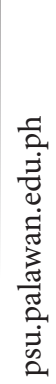 & 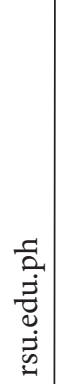 & 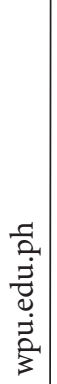 & 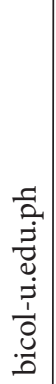 & 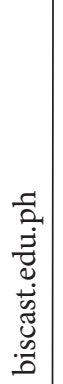 & 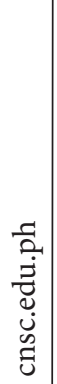 & 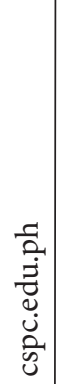 & 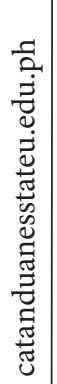 & 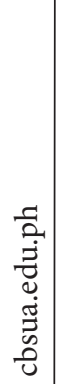 & 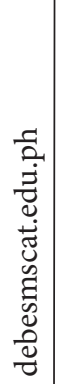 & 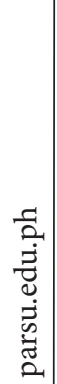 & 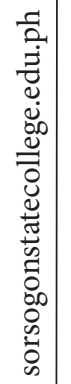 & 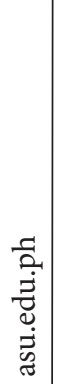 & 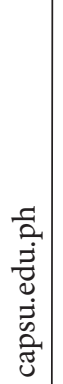 & 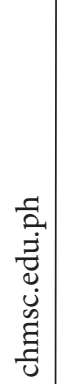 & 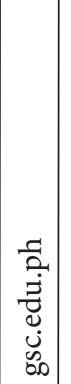 & 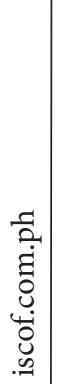 & 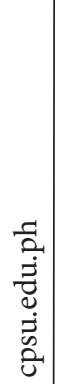 & 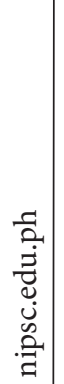 & 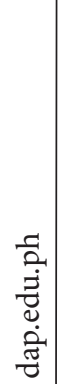 & 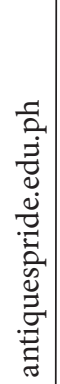 & 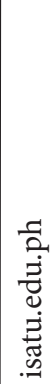 & 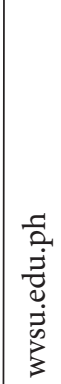 \\
\hline
\end{tabular}




\begin{tabular}{|c|c|c|c|c|}
\hline ТТУ४Н $\Lambda$ O & 2 & $\bar{\infty}$ & $\stackrel{0}{\infty}$ & \\
\hline 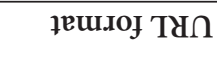 & $\stackrel{\circ}{\circ}$ & $\stackrel{\circ}{0}$ & $\stackrel{\circ}{0}$ & \\
\hline$. \partial \partial \| ! M_{L}$ & & & & \\
\hline 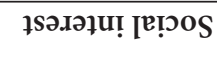 & $\stackrel{\circ}{\circ}$ & $\hat{b}$ & $\stackrel{\circ}{\circ}$ & \\
\hline 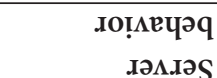 & $\stackrel{\circ}{\circ}$ & $\stackrel{\circ}{\circ}$ & $\stackrel{0}{\wedge}$ & \\
\hline 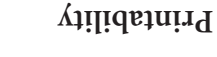 & ' & $\stackrel{0}{0}$ & $\stackrel{0}{0}$ & \\
\hline Kł!̣e[ndo & $\ln _{n \rightarrow 0}$ & กู่ & $\stackrel{\sim}{+}$ & \\
\hline 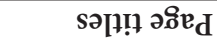 & $\stackrel{\circ}{\circ} \quad-1-1$ & $\stackrel{\circ}{\circ} \quad-1$ & $\stackrel{\circ}{\circ} \quad-1$ & \\
\hline ग!!१०W & $\stackrel{0}{\circ}$ & $\stackrel{0}{\circ}$ & $\because$ & ， \\
\hline Sถ̊ำ ำ & $\stackrel{\circ}{m}$ & $\stackrel{\circ}{i}$ & $\stackrel{\circ}{i}$ & . \\
\hline 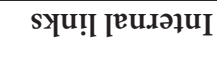 & $\stackrel{0}{0}^{0}$ & $\stackrel{\circ}{\circ}$ & 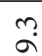 & . \\
\hline 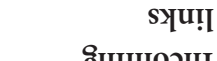 & $\stackrel{\circ}{\circ}$ & $\stackrel{\circ}{\circ}$ & $\stackrel{0}{\circ}$ & ， \\
\hline sวถిeuI & $\stackrel{\infty}{\infty}$ & $\stackrel{\infty}{\circ}$ & $\stackrel{\infty}{a}$ & , \\
\hline sภิu!peวH & $\stackrel{\circ}{\circ}$ & $\stackrel{\circ}{\sim}$ & $\stackrel{0}{\circ}$ & ， \\
\hline ssəuY & $\because$ & $\stackrel{0}{\circ}$ & $\stackrel{0}{0}$ & ' \\
\hline 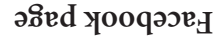 & & , & $\stackrel{2}{2}$ & . \\
\hline so!̣K[eut & $\because$ & $\stackrel{0}{0}$ & : & . \\
\hline 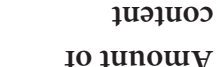 & $\stackrel{\circ}{\infty}$ & $\stackrel{\infty}{\infty}$ & $\stackrel{\infty}{\infty}$ & . \\
\hline 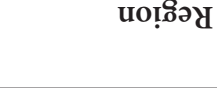 & $n$ & $n$ & $n$ & $n$ \\
\hline 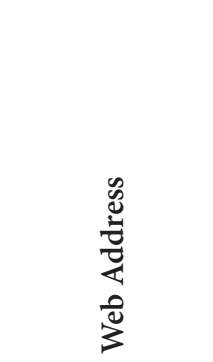 & 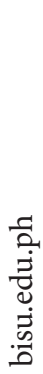 & 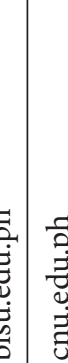 & 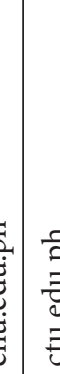 & 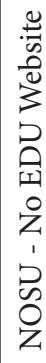 \\
\hline
\end{tabular}

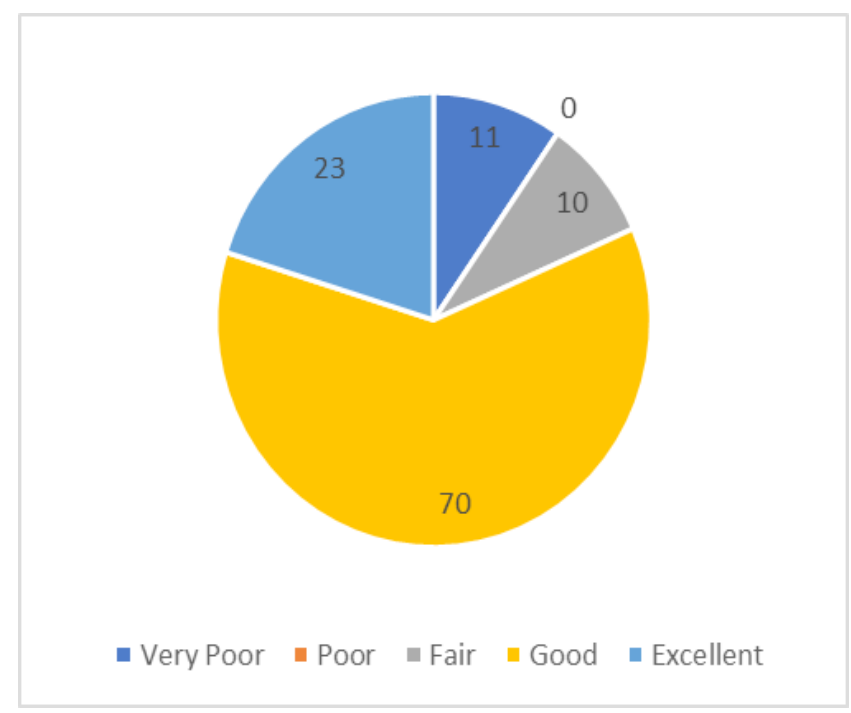

Figure 1. Total number of SUC with Excellent Web Rating with a total of 23 .

university should designate a University or College Web Administrator to manage the website. This would enable the University in the world of education, to publish their school and to offer related information to their user. It is also noticeable that University already implemented blended learning approach thru the use of free online tools such as Edmodo and Google Classroom as seen in the links of their respective website. The University of the Philippines still the most visible institution with an excellent virtual learning environment platform that consists of open source LMS such as Moodle. It is recommended that SUC all over the Philippines should benchmark on this model university.

Based on the result of the last batch of the list of SUC website as shown in Table 3, For Region 8, Visayas State University Ranked first with a Rating Score of 8.9. Zamboanga City State Polytechnic College was the first in Region 9 with an overall rating score of 7.6. While in Region 10, MSU - Iligan Institute of Technology ranked first with an overall rating score of 8.3. In Region 11, the Southern Philippines Agri-Business and Marine and Aquatic School of Technology are the first with an overall score of 8.4. In region 12, the Cotabato State University ranked first with an overall rating score of 7.8. In the ARMM Region, Mindanao State University is the first with an overall rating score of 7.8. Lastly, Surigao State College of Technology is the top in Region 13 with a rating score of 7.2.

In the third batch of the result, Visayas State University is the highest with a rating score of 8.9, 


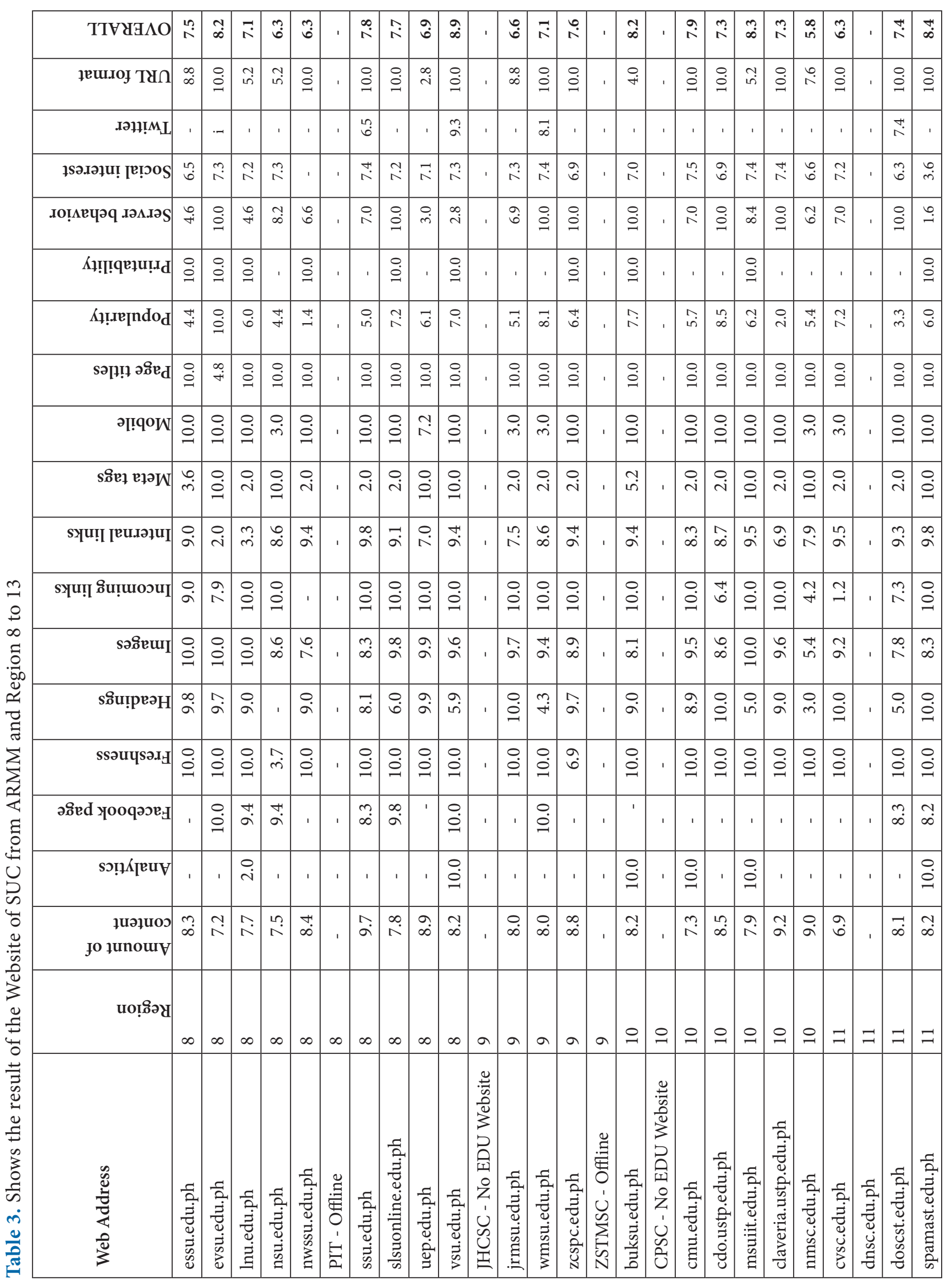




\begin{tabular}{|c|c|c|c|c|c|c|c|c|c|c|c|c|c|c|c|}
\hline ТТУУ目 & $\stackrel{0}{\Lambda}$ & $\stackrel{\infty}{\wedge}$ & 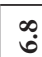 & $\stackrel{\infty}{i}$ & $\stackrel{?}{n}$ & ' & $\vec{b}$ & $\stackrel{\infty}{\wedge}$ & $\stackrel{+}{n}$ & $q$ & & & in & $\stackrel{\circ}{n}$ & $\stackrel{?}{\pi}$ \\
\hline деш.коу Т8 & $\stackrel{0}{0}$ & $\begin{array}{l}\infty \\
\infty \\
\infty\end{array}$ & $\begin{array}{l}\infty \\
\infty\end{array}$ & $\stackrel{\circ}{\stackrel{\circ}{9}}$ & $\stackrel{\circ}{0}$ & ' & $\stackrel{0}{\stackrel{9}{9}}$ & $\stackrel{0}{\sim}$ & $\stackrel{\circ}{\ominus}$ & $\stackrel{+}{+}$ & & & กี & กี่ & $\stackrel{\circ}{\oplus}$ \\
\hline 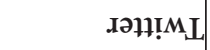 & $\stackrel{\infty}{\sim}$ & & & & ' & ' & ' & ' & & & & & , & & \\
\hline 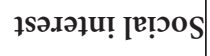 & $\stackrel{\infty}{n}$ & ir & iี & : & $F$ & ' & $\begin{array}{l}\overrightarrow{0} \\
\dot{0}\end{array}$ & $\stackrel{\infty}{\sim}$ & 8 & & & & $\hat{\sigma}$ & in & ?ु. \\
\hline 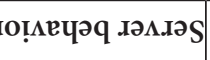 & \begin{tabular}{|l|} 
\\
+ \\
+
\end{tabular} & 它 & $\stackrel{0}{6}$ & $\stackrel{0}{0}$ & فe & ' & $\stackrel{0}{0}$ & : & $\stackrel{\circ}{i}$ & $\underset{\infty}{\infty}$ & & & $\underset{\infty}{\infty}$ & $\stackrel{i}{i}$ & $\stackrel{\circ}{\wedge}$ \\
\hline 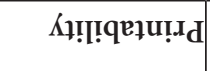 & 0 & $\stackrel{\circ}{\stackrel{\dot{\varphi}}{1}}$ & $\stackrel{\circ}{\ominus}$ & ' & ' & ' & $\stackrel{0}{9}$ & $\stackrel{\circ}{\varrho}$ & $\stackrel{\circ}{\ominus}$ & & & & ' & $\stackrel{\circ}{\oplus}$ & $\stackrel{\circ}{9}$ \\
\hline Kұ!̣e[ndo & $\stackrel{\digamma}{+}$ & $\stackrel{\infty}{i}$ & $\vec{i}$ & 蚝 & in & , & $\exists$ & $\omega_{\infty}^{10}$ & $\vec{m}$ & $\stackrel{\circ}{i}$ & & & $\stackrel{\circ}{\circ}$ & : & $\stackrel{\circ}{+}$ \\
\hline 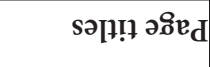 & $\stackrel{\circ}{\ominus}$ & $\stackrel{\circ}{\stackrel{9}{9}}$ & $\stackrel{\circ}{9}$ & $\stackrel{\circ}{9}$ & $\stackrel{\circ}{\ominus}$ & ' & $\stackrel{0}{\circ}$ & $\stackrel{\circ}{0}$ & $\stackrel{0}{\dot{\Theta}}$ & $\stackrel{0}{\circ}$ & & & $\stackrel{\circ}{\circ}$ & $\stackrel{0}{\circ}$ & $\stackrel{\circ}{\circ}$ \\
\hline ә!!१०М & $\stackrel{\circ}{\stackrel{9}{9}}$ & $\stackrel{0}{\dot{9}}$ & $\because$ & $\stackrel{\circ}{\dot{m}}$ & $\stackrel{\circ}{m}$ & ' & $\stackrel{0}{0}$ & Fi & $\stackrel{0}{\stackrel{9}{9}}$ & $\stackrel{\circ}{\dot{m}}$ & ' & & $\stackrel{\circ}{\dot{m}}$ & $\stackrel{0}{0}$ & $\stackrel{\circ}{\circ}$ \\
\hline 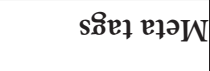 & $\stackrel{\circ}{i}$ & $\stackrel{\circ}{\stackrel{0}{9}}$ & $\because$ & $\stackrel{\circ}{i}$ & $\stackrel{0}{0}$ & ' & $\stackrel{i}{i}$ & $\stackrel{\circ}{\circ}$ & $\stackrel{\circ}{i}$ & $\stackrel{\circ}{i}$ & ' & & $\stackrel{\circ}{i}$ & $\stackrel{0}{\circ}$ & $\stackrel{\circ}{i}$ \\
\hline 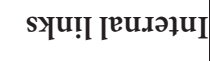 & $\because$ & $\stackrel{\circ}{\circ}$ & $\mathscr{\infty}_{0}^{\infty}$ & $\stackrel{0}{\circ}$ & $\stackrel{8}{\circ}$ & . & $\underset{+}{\sim}$ & n. & ลู & $\stackrel{\sharp}{\ddot{n}}$ & ' & & -5 & $\ddot{b}$ & $\underset{\infty}{1}$ \\
\hline 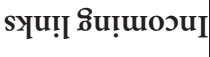 & $\because$ & $\stackrel{.}{\circ}$ & $\stackrel{\circ}{\circ}$ & $\stackrel{0}{0}$ & $\because$ & ' & $\stackrel{\circ}{\dot{m}}$ & $\stackrel{0}{\circ}$ & $\ddot{0}$ & $\stackrel{\sim}{\longrightarrow}$ & ' & & $\because$ & $\stackrel{0}{\circ}$ & $\stackrel{\circ}{\circ}$ \\
\hline sə8̊euा & $\stackrel{n}{~}$ & $\mid \begin{array}{l}10 \\
\infty\end{array}$ & $\vec{a}$ & $\stackrel{0}{0}$ & $a$ & , & aे & $\begin{array}{l}0 \\
\infty \\
\infty\end{array}$ & $\stackrel{\infty}{\infty}$ & $\underset{\infty}{\infty}$ & 1 & & $\underset{\infty}{0}$ & $\stackrel{\infty}{a}$ & $\vec{a}$ \\
\hline s.ิu!peəH & $\stackrel{\circ}{\circ}$ & $\stackrel{0}{\circ}$ & in & ' & in & & $\begin{array}{l}0 \\
\infty \\
\infty\end{array}$ & $\stackrel{0}{\circ}$ & $\stackrel{m}{\infty}$ & $a$ & ' & & in & $\ddot{H}$ & $\stackrel{\circ}{\circ}$ \\
\hline ssəuપsә., & $\ddot{0}$ & $\stackrel{0}{0}$ & $\because$ & $\stackrel{9}{n}$ & $\stackrel{\circ}{\circ}$ & ' & $\because$ & $\because$ & $\ddot{0}$ & $\begin{array}{l}\infty \\
\infty \\
\emptyset\end{array}$ & ' & & $\because$ & $\stackrel{0}{0}$ & $\stackrel{\infty}{+}$ \\
\hline 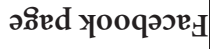 & , & , & , & 1 & & & ' & $\stackrel{\circ}{\circ}$ & ' & ' & ' & & ' & ' & 1 \\
\hline so!ฺK[euV & . & ' & ' & ' & $\because$ & & ' & $\stackrel{\circ}{i}$ & ' & ' & ' & & $\stackrel{0}{0}$ & . & , \\
\hline 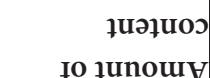 & $\ddot{0}$ & $\vec{a}$ & $=$ & , & $\stackrel{m}{\infty}$ & & $\stackrel{\sim}{\sim}$ & $\stackrel{0}{\infty}$ & $\underset{\infty}{\infty}$ & $\underset{\infty}{+!}$ & ' & & $\stackrel{\infty}{\wedge}$ & $\stackrel{n}{\sim}$ & $\underset{\infty}{\infty}$ \\
\hline uo!̣ßoิ & $=$ & $\simeq$ & $\mathcal{I}$ & $\simeq$ & $\simeq$ & 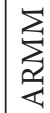 & 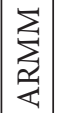 & 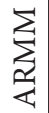 & 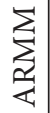 & $\sum_{\substack{4 \\
4}}$ & $\sum_{\substack{2 \\
<}}$ & $m$ & 2 & 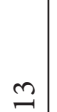 & $m$ \\
\hline 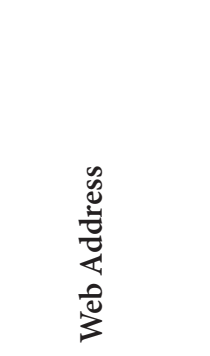 & 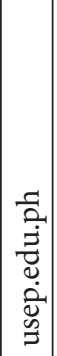 & 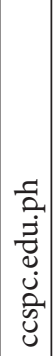 & 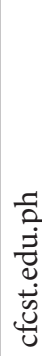 & 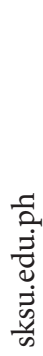 & 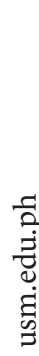 & 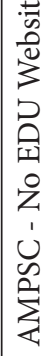 & 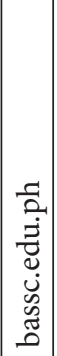 & 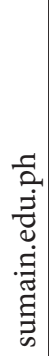 & 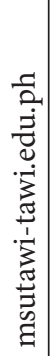 & 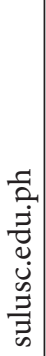 & 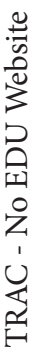 & 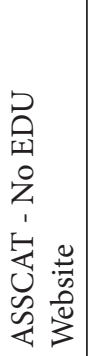 & 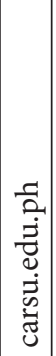 & 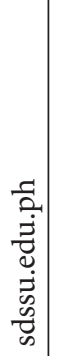 & 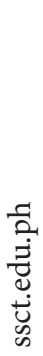 \\
\hline
\end{tabular}


Table 4. The overall ranking in the Philippines

\begin{tabular}{|c|c|c|c|c|}
\hline Name of SUC & Web Address & $\begin{array}{l}\stackrel{0}{0} \\
\stackrel{0}{0} \\
\simeq\end{array}$ & 胥 & $\stackrel{\mathscr{Z}}{\tilde{E}}$ \\
\hline $\begin{array}{l}\text { University of the } \\
\text { Philippines System }\end{array}$ & up.edu.ph & NCR & 8.9 & 1 \\
\hline Visayas State University & vsu.edu.ph & 8 & 8.9 & \\
\hline Capiz State University & capsu.edu.ph & 6 & 8.6 & 2 \\
\hline $\begin{array}{l}\text { Cebu Technological } \\
\text { University }\end{array}$ & ctu.edu.ph & 7 & 8.6 & \\
\hline Tarlac State University & tsu.edu.ph & 3 & 8.5 & 3 \\
\hline $\begin{array}{l}\text { Technological University } \\
\text { of the Philippines }\end{array}$ & tup.edu.ph & NCR & 8.4 & 4 \\
\hline $\begin{array}{l}\text { Mountain Province State } \\
\text { University }\end{array}$ & mpspc.edu.ph & CAR & 8.4 & \\
\hline $\begin{array}{l}\text { Pampanga State } \\
\text { Agricultural University }\end{array}$ & psau.edu.ph & 3 & 8.4 & \\
\hline $\begin{array}{l}\text { Southern Philippines } \\
\text { Agri-Business and } \\
\text { Marine and Aquatic } \\
\text { School of Technology }\end{array}$ & spamast.edu.ph & 11 & 8.4 & \\
\hline $\begin{array}{l}\text { Pangasinan State } \\
\text { University }\end{array}$ & psu.edu.ph & 1 & 8.3 & 5 \\
\hline $\begin{array}{l}\text { Iloilo State College of } \\
\text { Fisheries }\end{array}$ & iscof.com.ph & 6 & 8.3 & \\
\hline $\begin{array}{l}\text { Iloilo Science and } \\
\text { Technology University }\end{array}$ & isatu.edu.ph & 6 & 8.3 & \\
\hline $\begin{array}{l}\text { MSU - Iligan Institute of } \\
\text { Technology }\end{array}$ & msuiit.edu.ph & 10 & 8.3 & \\
\hline
\end{tabular}

making it the top in the whole SUC in the Philippines tied with the University of the Philippines. As shown in Table 4, the Pangasinan State University ranked $5^{\text {th }}$ in the whole nation with an overall rating of 8.3 with the descriptive equivalent of excellent.

This shows that Pangasinan State University website is excellent in the four categories which include accessibility, experience, marketing, and technology.

\section{Conclusion}

The result of the study shows that SUC website in the Philippines provided a satisfying result. The Top 5 institutional website based on the testing tool was known as the institution with a high number of Center of Excellence and Center of Development. It is concluded that Pangasinan State University is doing well in web rankings, the website of the institution provides the best result in the benchmarking compared to the most number of SUC website in the country. It is recommended that in the future study, the data should be utilized for more analyzation. While some of the SUC websites which don't have an institutional website should create one for the system, while other SUC website with GOV.PH domain is suggested to shift in EDU.PH domain for recognition.

\section{Acknowledgement}

The author thanks the following who made this research a success. Firstly, to the President of the Pangasinan State University for always motivating to do the research; Dr. Nova Arquillano who always mentored the author in this research journey. The author also thanks Executive Director for Research, Dr. Francis Albert Argente, the OUS Executive Director, Dr. Valentin Calpo, and Dr. Phillip Queroda.

\section{References}

1. Thabtah F, Abdelhamid N. Ranking and grouping website. 2016 7th International Conference on Computer cience and Information Technology (CSIT); 2016. p. 1-5.

2. Zilincan J. Search engine optimization. CBU International Conference Proceedings. 2015; 3:1-506.

3. Tomasi S, Li X. Influences of search engine optimization on performance of SMEs: A qualitative perceptive. Journal of Electronic Commerce in Organizations. 2015; 13(1):27-49. https://doi.org/10.4018/jeco.2015010103

4. The Local Government Code of the Philippines [Internet]. [cited 2018]. Available from: http://www.chanrobles.com/ localgov3.htm.

5. Sarlis AS, Drivas IC, Sakas DP. Implementation and dynamic simulation modeling of search engine optimization processes. Improvement of Website Ranking. Strategic Innovative Marketing; 2017. p. 437-43.

6. Pan $B$. The power of search engine ranking for tourist destinations. Tourism Management. 2015; 47:79-87. https://doi. org/10.1016/j.tourman.2014.08.015

7. About Nibbler [Internet]. [cited 2018 May 6]. Available from: http://nibbler.silktide.com/en_US/about.

8. Patel PR, Patel KM. To study effect of social media to improve website ranking. International Journal for Scientific Research and Development. 2014; 2(2):345-8.

9. Zilincan J. Search engine optimization. CBU International Conference Proceedings. 2015; 3:1-506. 
10. Canziani BF, WelshDH. Website quality for SME wineries: Measurement insights. Journal of Hospi-tality and Tourism Technology. 2016; 7(3):266-80. https://doi.org/10.1108/ JHTT-02-2016-0009

11. LIST: State colleges and universities covered by free tuition law [Internet]. [cited 2017 Aug 04]. Available from: https://www.rappler.com/nation/177684-list-statecolleges-universities-free-tuition.
12. Centers of Excellence and Centers of Development [Internet]. [cited 2018 May 6]. Available from: http:// ched.gov.ph/centers-excellence-centers-developmentcoescods.

13. Taddeo C, Barnes A. The school website: Facilitating communication engagement and learning. Brit-ish Journal of Educational Technology. 2016; 47(2):421-36. https://doi. org/10.1111/bjet.12229 